\title{
Role of apoptosis in the hypoxic regulation of human embryonic stem cells
}

\author{
Authors: DH Chettiar, ${ }^{\mathrm{A}}$ SA Arthur, ${ }^{\mathrm{A}}$ KL Parry ${ }^{\mathrm{A}}$ and FD Houghton ${ }^{\mathrm{A}}$
}

A

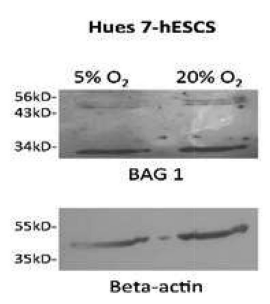

B

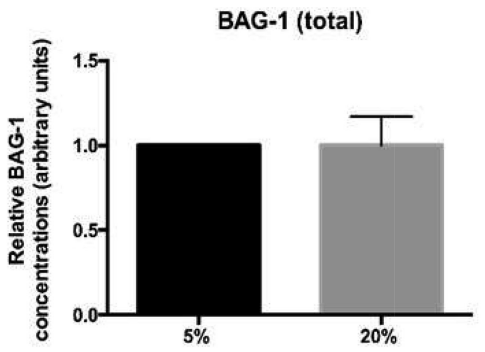

Oxygen concentration
C

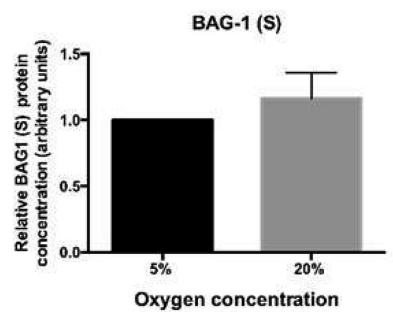

Oxygen concentration

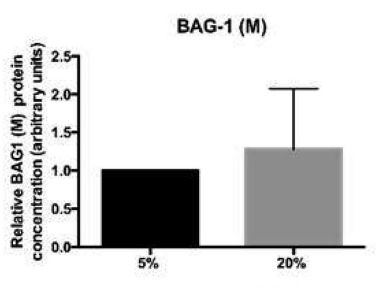

Oxygen concentration



Oxygen concentration
Fig 1. BAG1 expression is not significantly different in hESCs cultured at hypoxia compared with hESCs maintained at atmospheric oxygen. A) Representative blot where data were normalised to $\beta$-actin and to 1 for expression at $5 \% \mathrm{O}_{2}$. B) No significant difference in total BAG1 expression. C) No significant difference in BAG1 S, M and L expression $(n=5)$. Bars represent mean \pm standard error of the mean.

\section{Background}

Human embryonic stem cells (hESCs) are pluripotent cells that originate from the inner cell mass of a blastocyst. Their limitless differentiation capacity and ability to self-renew, also known as 'stemness', give them great potential in regenerative medicine. However, hESCs need to be maintained as a highly proliferative and pluripotent population to be used in clinical application. ${ }^{1}$ Much evidence suggests that a hypoxic (5\% oxygen) culture is more beneficial in maintaining hESC stemness than atmospheric (20\% oxygen) cultures. ${ }^{1-4}$ Studies have shown a higher expression of transcription factors NANOG, SOX2 and OCT-4, which regulate hESC pluripotency, and a lower rate of apoptosis in hESCs cultured at 5\% oxygen compared with those maintained at $20 \%$ oxygen. This is perhaps not surprising since the pre-implantation embryo develops in a hypoxic reproductive tract, ranging from $1.5-5.3 \%$ oxygen concentration. ${ }^{5}$

Authors: ${ }^{A}$ Centre for Human Development, Stem Cells and Regeneration, Southampton, UK
However, the regulation of apoptosis in hESCs under hypoxia has been largely overlooked. ${ }^{6}$ BCL2 anthogene 1 (BAG1) is a recently discovered multifunctional protein that is involved in many cellular processes including apoptosis, transcription, proliferation, cell signalling and differentiation. ${ }^{7}$ BAG1 expression has been shown to be upregulated in mesenchymal and trophoblastic cells under hypoxia, suggesting that its expression may be regulated by environmental oxygen tensions. ${ }^{7}$ BAG1 is overexpressed in many cancers, suggesting that it may contribute to cancer pathogenesis by increasing resistance to apoptosis. ${ }^{8}$ BAG1 knockout experiments in mice have demonstrated its requirement for the survival and differentiation of haematopoietic and neuronal cells. ${ }^{8}$

This study therefore aimed to investigate whether BAG1 has a role in regulating the increased proliferation of hESCs observed at hypoxic conditions and whether it has an effect on hESC self-renewal.

\section{Methods and results}

Immunocytochemistry showed that BAG1 is expressed in hESCs, suggesting that it may have a function in the 
A

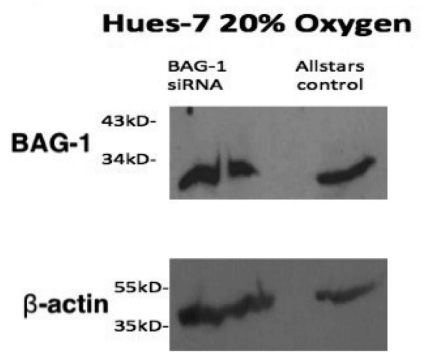

Fig 2. BAG1 does not regulate OCT-4 expression. Human embryonic stem cells cultured at $20 \% \mathrm{O}_{2}$ were transfected with either an Allstars control small interfering ribonucleic acid (siRNA) or BAG1 siRNA. A) BAG1 expression in human embryonic stem cells transfected with BAG1 siRNA was significantly reduced $(p=0.0435$; $n=3)$. B) No significant difference in OCT-4 expression in BAG1 siRNAtransfected human embryonic stem cells $(n=3)$. Data were normalised to $\beta$-actin and to 1 for control siRNA-treated cells. Bars represent mean \pm standard error of the mean.



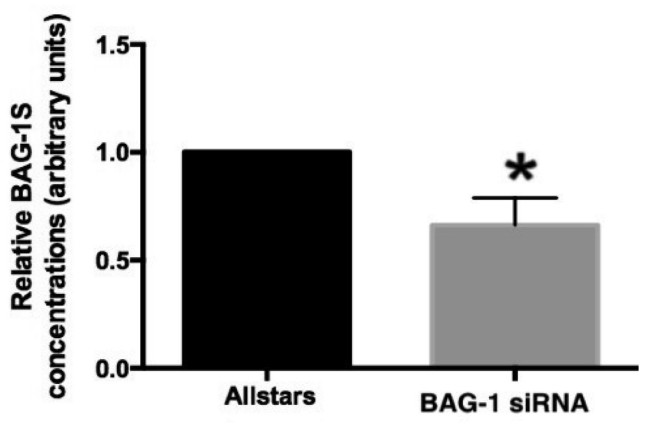

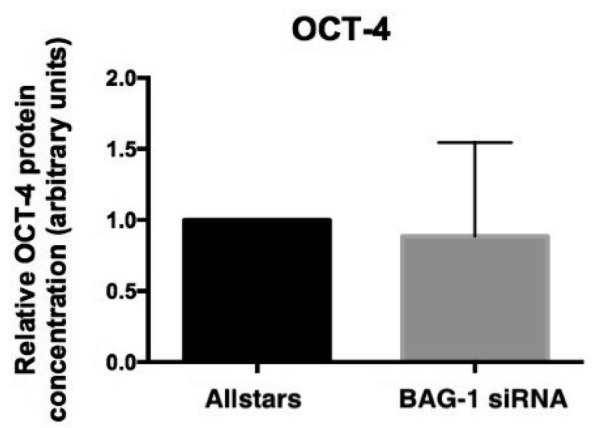

maintenance of hESC stemness. It showed that BAG1 expression was localised to both the cytoplasm and nucleus in hESCs cultured at both $5 \%$ and $20 \%$ oxygen. But Western blotting showed no significant difference in BAG1 expression between hESCs cultured at 5\% oxygen tension and those maintained at $20 \%$ oxygen tension (Fig 1). This suggests that BAG1 expression in hESCs is not dependent on environmental oxygen tensions.

BAG1 was also silenced in hESCs cultured at $20 \%$ oxygen using small interfering ribonucleic acid, which showed no significant effect on OCT-4 expression in hESCs compared with negative control (Fig 2). This suggests that BAG1 does not regulate hESC self-renewal. A recent publication by Tang et al (2017) supported this finding in murine embryonic stem cells (mESCs), and reported that BAG1 homozygous knockout mESCs had a normal karyotype and maintenance of pluripotency. ${ }^{9}$

\section{Conclusions}

The present study therefore shows that BAG1 does not play a role in the hypoxic response of hESCs and that perhaps other pro-survival proteins may be involved. Gene expression profiling comparing anti-apoptotic gene expression, including BAG1, between hESCs and cancer cell lines showed that only two anti-apoptotic factors, BCL10 and BIRC5 (survivin), are preferentially expressed in hESCs. ${ }^{10}$ Future work should therefore be directed to elucidating the roles of other antiapoptotic proteins in hESCs.

\section{Conflicts of interest}

None declared.

\section{References}

1 Forristal CE, Christensen DR, Chinnery FE et al. Environmental oxygen tension regulates the energy metabolism and self-renewal of human embryonic stem cells. PLoS One 2013;8:e62507.

2 Forristal CE, Wright KL, Hanley NA, Oreffo ROC, Houghton FD. Hypoxia inducible factors regulate pluripotency and proliferation in human embryonic stem cells cultured at reduced oxygen tensions. Reproduction 2010;139:85-97.

3 Christensen DR, Calder PC, Houghton FD. GLUT3 and PKM2 regulate OCT4 expression and support the hypoxic culture of human embryonic stem cells. Sci Rep 2015;5:17500.

4 Houghton FD. Energy metabolism of the inner cell mass and trophectoderm of the mouse blastocyst. Differentiation 2006;74:118.

5 Hu CJ, Wang LY, Chodosh LA, Keith B, Simon MC. Differential roles of hypoxia-inducible factor $1 \alpha$ (HIF- $1 \alpha)$ and HIF- $2 \alpha$ in hypoxic gene regulation. Mol Cell Biol 2003;23:9361-74.

6 Bergmann A, Steller H. Apoptosis, stem cells, and tissue regeneration. Sci Signal 2010;3:re8.

7 Takayama S, Krajewski S, Krajewska M et al. Expression and location of Hsp70/Hsc-binding anti-apoptotic protein BAG-1 and its variants in normal tissues and tumor cell lines. Cancer Res 1998;58:3116-31.

8 Prinsloo E, Setati MM, Longshaw VM, Blatch GL. Chaperoning stem cells: a role for heat shock proteins in the modulation of stem cell self-renewal and differentiation? Bioessays 2009;31:370-7.

9 Tang CC, Shan LP, Wang WM, Lu G, Tare RS, Lee KKH. Generation of a Bag1 homozygous knockout mouse embryonic stem cell line using CRISPR/Cas9. Stem Cell Res 2017;21:29-31.

10 Bader AM, Klose K, Bieback K et al. Hypoxic preconditioning increases survival and pro-angiogenic capacity of human cord blood mesenchymal stromal cells in vitro. PLoS One 2015;10:e0138477. 